\title{
EFEITO AGUDO DO ULTRASSOM TERAPÊUTICO CONTÍNUO NA TEMPERATURA DA PELE, EM REGIÃO DE PONTO GATILHO: RELATO DE CASO
}

\author{
Michelle Borges Nery Ferreira ${ }^{1}$, Cleber Souza de Jesus ${ }^{2}$
}

1. Fisioterapeuta Especialista em Dor, Belo Horizonte, Brasil.

2. Departamento de Saúde II, Universidade Estadual do Sudoeste da Bahia. Jequié, Brasil.

\begin{abstract}
RESUMO
O ultrassom terapêutico é utilizado no tratamento de ponto gatilho miofascial e a termografia pode ser utilizada como ferramenta auxiliar no diagnóstico e no acompanhamento desses casos. Dessa forma, este estudo teve por objetivo avaliar a resposta aguda do ultrassom contínuo no tratamento de ponto gatilho miofascial por meio da termografia, em um paciente do sexo masculino, sedentário, com 41 anos de idade e sem comorbidades. Na avaliação termográfica foi identificado um ponto gatilho em região de trapézio do lado direito, cuja temperatura excedia em $0,4^{\circ} \mathrm{C}$ o lado contralateral. Em seguida, foi aplicado ultrassom terapêutico contínuo por quatro minutos, frequência de $3 \mathrm{Mhz}$ e intensidade de $1,4 \mathrm{~W} / \mathrm{cm}^{2}$. Após conduta terapêutica, foi realizado seguimento da região de interesse por meio da termografia com imagens a cada 2 minutos. Imediatamente após a aplicação, a temperatura local foi elevada em $4,4^{\circ} \mathrm{C}$ com posterior redução até o $7^{\circ}$ minuto. A partir do $9^{\circ}$ minuto, foi registrado um ligeiro aumento da temperatura da pele no local da intervenção, e, a partir de então, a temperatura se manteve praticamente constante até os minutos finais de acompanhamento. Houve redução da intensidade da dor e da percepção sensorial da localização do ponto gatilho. Este relato de caso revela que a termografia possui grande potencial de aplicação tanto no auxílio diagnóstico quanto no monitoramento da evolução do tratamento.

Palavras-Chave: Síndromes da dor miofascial, Terapia por ultrassom, Termografia.
\end{abstract}

\begin{abstract}
Therapeutic ultrasound is commonly used in the treatment of myofascial trigger point and thermography can be used as an auxiliary tool in the diagnosis and monitoring of these cases. Thus, this study aimed to evaluate the acute response of continuous ultrasound in the treatment of myofascial trigger point through thermography. In the thermographic evaluation, two trigger points were identified in the trapezius region on the right side, whose temperature exceeded the contralateral side by $0.4^{\circ} \mathrm{C}$. Then, continuous therapeutic ultrasound was applied for four minutes, frequency of $3 \mathrm{Mhz}$ and intensity of $1.4 \mathrm{~W} / \mathrm{cm}^{2}$. After the therapeutic approach, the region of interest was followed by thermography with images every 2 minutes. Immediately after application, the local temperature was raised by $4.4^{\circ} \mathrm{C}$ with a subsequent reduction until the 7 th minute. From the 9 th minute on, a slight increase in skin temperature at the intervention site was recorded, and from then on, the temperature remained practically constant until the final minutes of follow-up. There was a reduction in pain intensity and sensory perception of the trigger point location. This case report reveals that thermography has great application potential both in aiding diagnosis and in monitoring the evolution of treatment.
\end{abstract}

Key Words: Myofascial Pain Syndromes, Ultrasonic Therapy, Thermography.

DOI: http://dx.doi.org/10.18073/pajmt.2020.7.003 


\section{INTRODUÇÃO}

Nos últimos anos, o aumento no tempo de uso de tela tornou-se evidente. A utilização do computador, por grandes períodos, e a manutenção de posturas inadequadas favorecem a ocorrência dos distúrbios musculoesqueléticos ${ }^{1}$.

A sobrecarga biomecânica do tecido muscular está frequentemente relacionada à manutenção da postura estática, o que resulta no aumento da atividade do músculo com consequente formação do ciclo espasmo-dor-espasmo ${ }^{2}$. Esse ciclo leva à redução do fluxo sanguíneo e do aporte de oxigênio favorecendo a ocorrência de pontos gatilhos $^{3}$. 0 trapézio superior e o infraespinal são os músculos mais acometidos com a incidência de pontos gatilhos em pacientes com dor cervical crônica não específica, e muitos destes pacientes, não apresentam sinais de alteração em exames por ressonância magnética ${ }^{2}$.

O ponto gatilho miofascial (PGM) é caracterizado como um nódulo palpável hipersensível em uma banda de tensão musculoesquelética. Pode ser classificado como ativo, quando gera sintomas, ou latente, quando está assintomático ${ }^{4,5}$. O PGM doloroso à palpação pode causar sensibilidade ou dor referida, disfunção motora e fenômenos autonômicos ${ }^{4}$. Aqueles do tipo latente são de elevada incidência em músculos esqueléticos de indivíduos adultos ${ }^{5}$.

Estudos relacionam o surgimento de ponto gatilho miofascial com alterações na atividade e biomecânica do tecido muscular, além de repercussões no metabolismo e no fluxo sanguíneo local ${ }^{6}$, mas os mecanismos envolvidos na gênese do ponto gatilho não estão totalmente esclarecidos ${ }^{7}$. Na prática clínica, a palpação é o recurso mais utilizado no diagnóstico do ponto gatilho, conforme diversos estudos ${ }^{8,9}$. Entretanto, essa técnica apresenta várias limitações como pouca sensibilidade e especificidade, fato que pode prejudicar o diagnóstico, e sobretudo a localização de maneira adequada ${ }^{10}$. Outros métodos também têm sido utilizados no diagnóstico de ponto gatilho como a ultrassonografia, a eletromiografia e a termografia ${ }^{6}$. Em 2007, Brioschi e colaboradores ${ }^{11}$ relataram que a termografia é o único método diagnóstico que consegue mostrar, de forma objetiva, os pontos gatilhos, que podem ser vistos como pontos hiperradiantes. Além disso, a imagem do ponto gatilho é típica e a alteração de padrão de radiação cutânea produzida por esses pontos consegue ser captada e mensurada pelos sensores infravermelhos.

Dentre os diversos tratamentos para o ponto gatilho miofascial destacam-se os exercícios terapêuticos, alongamentos, terapia manual, ultrassom terapêutico, laser e agulhamento seco. 0 ultrassom terapêutico (UST) é um recurso não invasivo, de efeitos térmicos e biofísicos, muito utilizado na dor miofascial $^{12}$. Estudos mostram que o ultrassom terapêutico contínuo pode reduzir o quadro álgico através do aumento da temperatura e do metabolismo local, bem como através da geração de vasodilatação ${ }^{13}$.

Apesar dos crescentes estudos sobre a aplicabilidade da termografia no diagnóstico de pontos gatilhos miofasciais, os estudos encontrados, considerando pontos latentes, foram escassos. Levando-se em consideração a possibilidade de utilização da termografia no acompanhamento dos pacientes durante a evolução do tratamento da dor miofascial e a incidência desse tipo de disfunção associada a pontos gatilhos miofasciais latentes, este estudo teve por objetivo avaliar a resposta aguda do 
ultrassom terapêutico contínuo no tratamento de ponto gatilho miofascial, por meio da termografia.

\section{METODOLOGIA}

\section{Informações do paciente}

Este é um estudo do tipo relato de caso conduzido com paciente do sexo masculino de 41 anos de idade. 0 paciente não possui comorbidades diagnosticadas, nega uso de medicação e não pratica atividade física. 0 mesmo trabalha, em média, oito horas por dia na posição sentada, fazendo uso de computador. Realiza pequenas pausas durante a jornada de trabalho. Atualmente, durante alguns dias da semana, executa suas atividades em home office e considera que a disposição dos equipamentos em domicílio é menos adequada para sua postura de trabalho. Relata dor em região escapular há vários anos, com períodos de agudização.

\section{Achados Clínicos}

0 paciente relatou dor em ponto específico da região medial da escápula direita (terço superior), sem irradiação e com intensificação do quadro álgico ao carregar peso com o membro superior direito, trocar de travesseiro, ter noites mal dormidas e momentos de maior estresse. A sensação dolorosa diminui com massagem no local ou compressão nos pontos de dor. Ao exame físico, não apresentou alteração postural relevante, nem restrição da amplitude de movimento de coluna cervical ou ombros.

Para avaliar a intensidade da dor foi utilizada a Escala Analógica Visual (EVA), que se assemelha a uma régua, composta de números de 0 (zero) a 10 (dez). 0 paciente foi orientado que nota
0 equivale à ausência de dor e 10 corresponde à pior dor que ele já sentiu. Após aplicação da escala o paciente referiu dor de intensidade igual a 6 (seis) pontos.

\section{Avaliação diagnóstica por imagens ter- mográficas}

Para avaliar a temperatura da pele na região de interesse (ROI), as imagens térmicas foram captadas utilizando-se uma câmera térmica. A captação foi realizada em ambiente sem luz natural ou fluxo de ar direto sobre a região anatômica, a temperatura ambiente foi mantida em $22^{\circ} \mathrm{C}$ e umidade relativa do ar encontrava-se em $52 \%$.

0 paciente permaneceu sentado em uma cadeira sem apoio das costas e sem camisa, por vinte minutos, para garantir a termalização da região a ser captada. 0 local da dor relatada pelo paciente, foi verbalizado por ele e, na sequência, na região indicada, foi feita a marcação com lápis dermatográfico. Tal conduta foi realizada anteriormente ao início da termalização.

A câmera termográfica foi posicionada há setenta centímetros de distância do paciente para fins de captação das imagens. Foram coletadas dez imagens, sendo a primeira Pré-intervenção (Pre), uma logo após (P0), outra com um minuto após intervenção (P1), e as subsequentes a cada dois minutos até completar o tempo total de 15 (quinze) minutos (P3, P5, P7, P9, P11, P13 e P15). A câmera termográfica utilizada foi o modelo FLIR C5, resolução térmica de 19.200 pixels. 0 paciente foi orientado a manter a mesma postura durante a captação das imagens.

A Figura 1 demonstra a imagem termográfica do paciente com a localização das regiões de interesse bilateralmente demarcadas. 


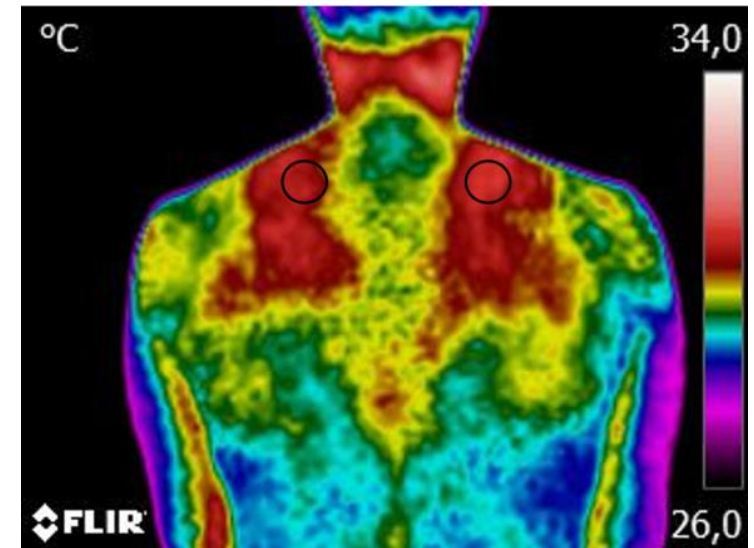

Figura 1. Imagem termográfica com localização das regiões de interesse (ROIs), direita e esquerda, anterior à aplicação do ultrassom terapêutico.

\section{Intervenção terapêutica}

Após a captação da imagem térmica Pré-intervenção (Pré) foi dado início à aplicação do ultrassom terapêutico, cujo equipamento é modelo Sonopulse III Ibramed - 1Mhz e 3Mhz. O protocolo de tratamento seguiu os seguintes parâmetros: modo de aplicação contínuo, realização de movimento circulares, tempo de aplicação de quatro minutos, com frequência de $3 \mathrm{Mhz}$ e intensidade de $1,4 \mathrm{~W} / \mathrm{cm}^{2}$. 0 cabeçote foi acoplado à pele do paciente com aplicação de gel à base de água. A área de aplicação tinha aproximadamente quatro centímetros de comprimento no sentido vertical e também no sentido horizontal. Imediatamente após a finalização da intervenção terapêutica, com uso de papel toalha de maneira bem superficial, foi retirado o gel da superfície da pele para não interferir nas análises das imagens.

\section{RESULTADOS}

A intensidade da dor avaliada pela EVA, no momento Pré-intervenção, foi de 6 pontos, caracterizada como de intensidade moderada. Quinze minutos após aplicação do US terapêutico na re- gião de PGM à direita, o paciente apontou intensidade da dor de nível 4 , ou seja, houve redução de 33,3\% na intensidade dolorosa. Foi verificada também uma redução da precisão de localização da resposta do paciente relativa aos pontos gatilhos.

Os parâmetros termográficos mensurados Pré-intervenção terapêutica mostraram valores de temperatura média mais elevados na região de interesse dos pontos gatilhos (ROI1d), uma diferença média de $0,4^{\circ} \mathrm{C}$ maior em região superomedial do trapézio direito comparativamente à mesma região em trapézio esquerdo.

A Figura 2 demonstra a evolução da temperatura média, nas regiões de interesse, bilateralmente. Durante todo o tempo, a temperatura média da pele sobre o trapézio direito, ficou mais elevada comparativamente à região de interesse do lado esquerdo (ROI1e) sendo essa diferença maior no momento PO, ou seja, imediatamente após a intervenção $\left(3,8^{\circ} \mathrm{C}\right)$. Após um minuto (P1), a diferença de temperatura entre as regiões de interesse reduziu para $1,6^{\circ} \mathrm{C}$, se mantendo em $1,9^{\circ} \mathrm{C}$ nos três momentos subsequentes (P3, P5 e P7). A partir do momento $\mathrm{P} 9$, a temperatura do lado direito se manteve praticamente constante até o momento P15.

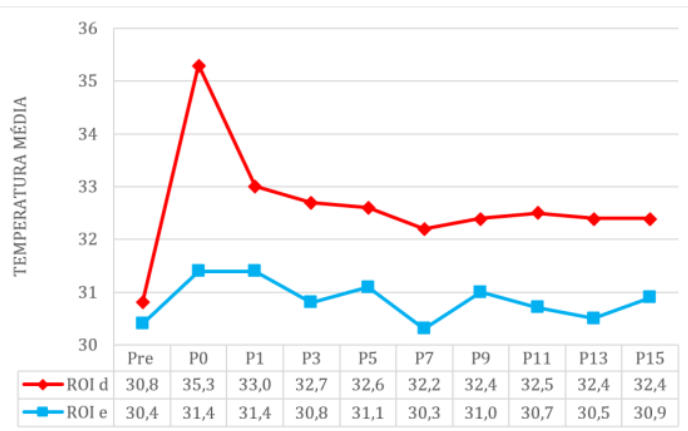

Figura 2. Temperaturas médias nas regiões de interesse de trapézio direito (ROI1d) e esquerdo (ROI1e) antes e após a aplicação do ultrassom terapêutico. 
Na Figura 3 são apresentadas as imagens termográficas capturadas no momento Pré-intervenção $(A)$ e nos minutos subsequentes à aplicação do ultrassom terapêutico até o $15^{\circ}$ minuto (J). $\mathrm{Na}$ análise qualitativa das imagens é possível visualizar que, no momento pós-intervenção imediata $(B)$, há maior intensidade de calor, e que a partir do $7^{\circ}$ minuto $(F)$ após intervenção há sinais de estabilização da temperatura no local com manutenção no tamanho da área.

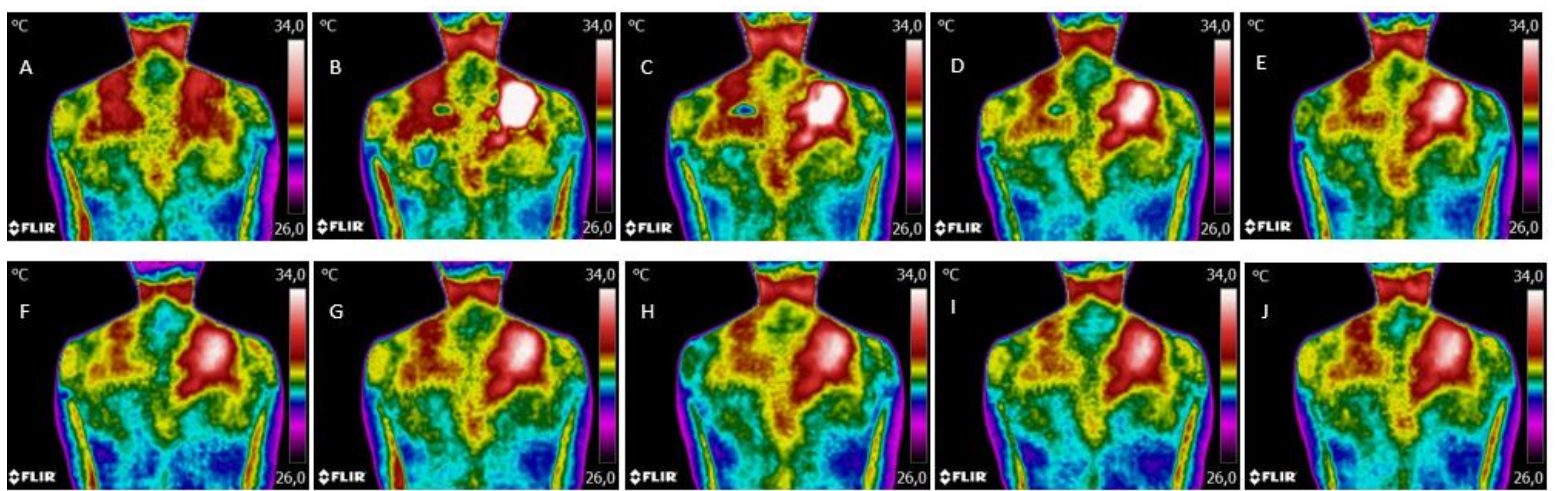

Figura 3. Evolução das imagens termográficas do momento Pré-intervenção $(A)$, imediatamente após aplicação do ultrassom terapêutico (B), 1 minuto (C), 3 minutos (D), 5 minutos (E), 7 minutos (F), 9 minutos (G), 11 minutos $(\mathrm{H})$, com 13 minutos $(\mathrm{I})$ e ao final de 15 minutos $(\mathrm{J})$.

\section{DISCUSSÃO}

O presente estudo avaliou, por meio de imagens termográficas, o efeito agudo do ultrassom terapêutico contínuo sobre ponto gatilho em região do músculo trapézio. Na avaliação termográfica inicial foi verificada uma assimetria térmica de $0,4^{\circ} \mathrm{C}$ entre a região de interesse do ponto gatilho miofascial e seu lado contralateral.

De acordo com a literatura, em situações normais, o padrão térmico esperado é de simetria e, a presença de alterações quantitativas e qualitativas nesse padrão tem sido relacionada à indicação de mudança do metabolismo e do processo hemodinâmico na região de interesse $(\mathrm{ROI})^{14}$. A termografia infravermelha é uma técnica utilizada como meio de diagnóstico de alterações fisiológicas e no acompanhamento de intervenções terapêuticas, através da mensuração da temperatura na superfície da pele $e^{6,9,15,16}$.
De acordo com o estudo de Cojocaru e colaboradores (2015) ${ }^{17}$, o mesmo padrão térmico do trigger point é encontrado em infecções localizadas da pele e, por essa razão, a termografia não deve ser o único recurso utilizado no diagnóstico da dor miofascial. Mas sua utilização como ferramenta auxiliar na avaliação e acompanhamento desse quadro clínico foi fortemente indicada. Em contrapartida, Brioschi e colaboradores (2007) ${ }^{11}$ afirmam que, através da termografia, é possível a captação e a mensuração das alterações dos padrões de radiações da pele geradas pelos PGMs. Outros estudos também consideram a termografia infravermeIha como um possível método de avaliação da dor miofascial, considerando as repercussões autonômicas e metabólicas envolvidas ${ }^{18}$.

Uma vez feita a localização dos pontos gatilhos foi realizada a aplicação do US terapêutico contínuo de baixa intensidade. Imediatamente após a interven- 
ção terapêutica e, com auxílio da termografia, observou-se a elevação de $4,4^{\circ} \mathrm{C}$ na temperatura da pele no local aplicado. Tal repercussão confirma os efeitos térmicos gerados por esse tipo de recurso terapêutico ${ }^{13}$.

Após o primeiro minuto, observou-se a queda significativa da temperatura, que se manteve em declínio durante os sete minutos iniciais após a intervenção. Esse comportamento pode ser explicado, em parte, pela vasodilatação decorrente do efeito térmico do ultrassom contínuo de baixa intensidade que eleva os níveis de óxido nítrico, ocasionando o aumento da vasodilatação, do fluxo sanguíneo, da oxigenação e do aporte de nutrientes ${ }^{13}$. Outro fator a ser considerado é o mecanismo de condução térmica realizada pela dissipação do calor local, mediante alteração no fluxo sanguíneo, na tentativa de normalizar a temperatura da pele.

A partir do $9^{\circ}$ minuto, a temperatura da pele começa a se elevar, de maneira discreta, e esse aumento acontece até o $15^{\circ}$ minuto, sendo que, durante todo esse período, a temperatura mantém valores praticamente constantes. Mesmo com essa elevação, a temperatura média da pele, ao final dos $15 \mathrm{mi}$ nutos, se mostra maior do que a temperatura Pré-intervenção, o que sugere que as repercussões da aplicação do ultrassom contínuo continuam presentes.

Após aplicação do ultrassom terapêutico e ao final dos 15 minutos, ao ser questionado sobre a localização dos pontos dolorosos, o paciente relatou redução tanto na percepção para localização, assim como na sensibilidade dolorosa à palpação dos pontos gatiIhos miofasciais. Portanto, conforme a literatura tem apresentado ${ }^{19}$, a utilização do US terapêutico é um importante recurso no alívio da dor em PGMs, por meio da redução da rigidez articular, de espasmos musculares, de dor e através do aumento do fluxo sanguíneo local $^{7,12,13}$. Alguns autores sugerem que a suprarregulação do óxido nítrico sintase, associada ao calor profundo, podem reduzir a sensibilidade dolorosa do ponto gatilho ${ }^{13}$.

\section{CONCLUSÃO}

Os resultados encontrados neste relato de caso confirmam diversos estudos sobre os efeitos térmicos do ultrassom terapêutico contínuo, bem como destacam a aplicabilidade da termografia como recurso de auxílio diagnóstico e acompanhamento. Entretanto, faz-se necessária a realização de outros estudos com a utilização de amostra mais numerosa, com a possibilidade de avaliação do efeito terapêutico do ultrassom contínuo por tempo maior, assim como acompanhamento do paciente com maior número de aplicações deste recurso.

\section{REFERÊNCIAS}

1. Andersen, J. H., Harhoff, M., Grimstrup, S., Vilstrup, I., Lassen, C. F., Brandt, L. P., Kryger, A. I., Overgaard, E., Hansen, K. D., \& Mikkelsen, S. Computer mouse use predicts acute pain but not prolonged or chronic pain in the neck and shoulder. Occupational and environmental medicine. 2008; 65(2): 126-131. https://doi.org/10.1136/oem.2007.033506

2. Ezzati, K., Ravarian, B., Saberi, A., Salari, A., Reyhanian, Z., Khakpour, M., \& Yousefzadeh Chabok, S. Prevalence of Cervical Myofascial Pain Syndrome and its Correlation with the Severity of Pain and Disability in Patients with Chronic Non-specific Neck Pain. The archives of bone and joint surgery. 2021; 9(2): 230-234.

https://doi.org/10.22038/abjs.2020.48697. 2415

3. Sadria, G., Hosseini, M., Rezasoltani, A., Akbarzadeh Bagheban, A., Davari, A., \& Seifolahi, A. A comparison of the effect of the active release and muscle energy techniques on the latent trigger points of the upper trapezius. Journal of bodywork and movement 
therapies. 2017; 21(4): 920-925. https://doi.org/10.1016/j.jbmt.2016.10.005

4. Do, T. P., Heldarskard, G. F., Kolding, L. T., Hvedstrup, J., \& Schytz, H. W. Myofascial trigger points in migraine and tension-type headache. The journal of headache and pain. 2018 ; 19(1): 84. https://doi.org/10.1186/s10194-018-09138

5. Moraska, A. F., Schmiege, S. J., Mann, J. D., Butryn, N., \& Krutsch, J. P. Responsiveness of Myofascial Trigger Points to Single and Multiple Trigger Point Release Massages: A Randomized, Placebo Controlled Trial. American journal of physical medicine \& rehabilitation. 2017; 96(9): 639-645. https://doi.org/10.1097/PHM.0000000000 000728

6. Dibai-Filho, A. V., Guirro, E. C., Ferreira, V. T., Brandino, H. E., Vaz, M. M., \& Guirro, R. R. Reliability of different methodologies of infrared image analysis of myofascial trigger points in the upper trapezius muscle. Brazilian journal of physical therapy. $2015 ; 19(2)$ : 122-128. https://doi.org/10.1590/bjptrbf.2014.0076

7. Xia, P., Wang, X., Lin, Q., Cheng, K., \& Li, X. Effectiveness of ultrasound therapy for myofascial pain syndrome: a systematic review and meta-analysis. Journal of pain research. 2017; 10 : 545-555. https://doi.org/10.2147/JPR.S131482

8. Bron, C., Dommerholt, J., Stegenga, B., Wensing, M., \& Oostendorp, R. A. High prevalence of shoulder girdle muscles with myofascial trigger points in patients with shoulder pain. BMC musculoskeletal disorders. 2011; 12 139. https://doi.org/10.1186/1471-2474-12-139

9. Magalhães, M. F., Dibai-Filho, A. V., de Oliveira Guirro, E. C., Girasol, C. E., de Oliveira, A. K., Dias, F. R., \& Guirro, R. R. Evolution of Skin Temperature after the Application of Compressive Forces on Tendon, Muscle and Myofascial Trigger Point. PloS one. 2015; $\quad$ 10(6): e0129034. https://doi.org/10.1371/journal.pone.0129034

10.Shah, J. P., Thaker, N., Heimur, J., Aredo, J. V., Sikdar, S., \& Gerber, L. Myofascial Trigger Points Then and Now: A Historical and Scientific Perspective. PM \& R: the journal of injury, function, and rehabilitation. 2015; 7(7): 746-761. https://doi.org/10.1016/j.pmrj.2015.01.024

11.Brioschi, M. L., Yeng, L. T., Pastor, E. M. H., Colman, D., Silva, F. M. M., \& Teixeira, M. J.
Documentation of myofascial pain syndrome with infrared imaging Marcos. Acta Fisiátrica. 2007; 14(1): 41-48.

12.Iter, L., Dilek, B., Batmaz, I., Ulu, M. A., Sariyildiz, M. A., Nas, K., \& Cevik, R. Efficacy of Pulsed and Continuous Therapeutic Ultrasound in Myofascial Pain Syndrome: A Randomized Controlled Study. American journal of physical medicine \& rehabilitation. 2015; 94(7): 547-554. https://doi.org/10.1097/PHM.0000000000 000210

13.Uddin, S., Komatsu, D. E., Motyka, T., \& Petterson, S. (2021). Low-Intensity Continuous Ultrasound Therapies-A Systemic Review of Current State-of-the-Art and Future Perspectives. Journal of clinical medicine. 2021; 10(12): https://doi.org/10.3390/jcm10122698

14. Haddad, D. S., Brioschi, M. L., \& Arita, E. S. Thermographic and clinical correlation of myofascial trigger points in the masticatory muscles. Dento maxillo facial radiology. 2012; $\quad 41(8)$ : 621-629. https://doi.org/10.1259/dmfr/98504520

15. de Meira, L. F., Krueger, E., Neves, E. B., Nohama, P., \& de Souza, M. A. Termografia na área biomédica. Pan American Journal of Medical Thermology. 2014; 1(1): 31-41.

16.Skorupska, E., Dybek, T., Rychlik, M., Jokiel, M., \& Dobrakowski, P. The Automatization of a New Thermography Method Using Invasive Nociceptive Stimulation to Confirm an Autonomic Phenomenon within a Trigger Point Referred Pain Zone. Brain sciences. 2021; 11(7): 893. https://doi.org/10.3390/brainsci11070893

17.Cojocaru, M. C., Cojocaru, I. M., Voiculescu, V. M., Cojan-Carlea, N. A., Dumitru, V. L., \& Berteanu, M. Trigger points--ultrasound and thermal findings. Journal of medicine and life. 2015; 8(3): 315-318.

18. Girasol, C. E., Dibai-Filho, A. V., de Oliveira, A. K., \& de Jesus Guirro, R. R. Correlation Between Skin Temperature Over Myofascial Trigger Points in the Upper Trapezius Muscle and Range of Motion, Electromyographic Activity, and Pain in Chronic Neck Pain Patients. Journal of manipulative and physiological therapeutics. 2018; 41(4): 350-357. https://doi.org/10.1016/j.jmpt.2017.10.009

19.Srbely, J. Z., Dickey, J. P., Lowerison, M., Edwards, M. A., Nolet, P. S., \& Wong, L. L. Stimulation of myofascial trigger points with ultrasound induces segmental antinociceptive effects: a randomized controlled study. Pain. 2008; 139(2): 260-266. https://doi.org/10.1016/j.pain.2008.04.009 